\title{
A!
}

This is an electronic reprint of the original article.

This reprint may differ from the original in pagination and typographic detail.

Dyadechkin, S.; Semenov, V. S.; Kallio, E.; Erkaev, N. V.; Alho, M.; Lammer, H.

\section{Global kinetic hybrid simulation for radially expanding solar wind}

Published in:

Journal of geophysical research: Space physics

DOI:

10.1002/2017JA023992

Published: 01/08/2017

Document Version

Publisher's PDF, also known as Version of record

Please cite the original version:

Dyadechkin, S., Semenov, V. S., Kallio, E., Erkaev, N. V., Alho, M., \& Lammer, H. (2017). Global kinetic hybrid simulation for radially expanding solar wind. Journal of geophysical research: Space physics, 122(8), 7854-7864. https://doi.org/10.1002/2017JA023992

This material is protected by copyright and other intellectual property rights, and duplication or sale of all or part of any of the repository collections is not permitted, except that material may be duplicated by you for your research use or educational purposes in electronic or print form. You must obtain permission for any other use. Electronic or print copies may not be offered, whether for sale or otherwise to anyone who is not an authorised user. 


\section{Journal of Geophysical Research: Space Physics}

\section{RESEARCH ARTICLE \\ 10.1002/2017JA023992 \\ Global kinetic hybrid simulation for radially expanding solar wind}

Key Points:

- A new global kinetic hybrid model

developed to investigate radially expanding solar wind

- The initially Maxwellian solar wind results in Parker's solar wind model type of acceleration

- The model can be used to analyze a non-Maxwellian solar wind and dynamical events

Correspondence to:

S. Dyadechkin,

sergey.dyadechkin@aalto

Citation:

Dyadechkin, S., V. S. Semenov, E. Kallio, N. V. Erkaev, M. Alho, and H. Lammer (2017), Global kinetic hybrid simulation for radially expanding solar wind, J. Geophys. Res. Space Physics, 122, 7854-7864, doi:10.1002/ 2017JA023992.

\section{Received 3 FEB 2017}

Accepted 7 JUL 2017

Accepted article online 13 JUL 2017

Published online 11 AUG 2017

๑2 2017. American Geophysical Union. All Rights Reserved.

\author{
S. Dyadechkin ${ }^{1,2}$, V. S. Semenov ${ }^{3}$, E. Kallio ${ }^{1}$, N. V. Erkaev ${ }^{4,5}$, M. Alho ${ }^{1}$, and H. Lammer ${ }^{2}$ \\ ${ }^{1}$ School of Electrical Engineering, Aalto University, Espoo, Finland, ${ }^{2}$ Space Research Institute, Austrian Academy of \\ Sciences, Graz, Austria, ${ }^{3}$ Earth Physics Department, St. Petersburg State University, St. Petersburg, Russia, ${ }^{4}$ Institute of \\ Computational Modelling, Siberian Branch of the Russian Academy of Sciences, Krasnoyarsk, Russia, ${ }^{5}$ Polytechnic \\ Institute, Department of Applied Mechanics, Siberian Federal University, Krasnoyarsk, Russia
}

Abstract We present the results of a 1-D global kinetic simulation of the solar wind in spherical coordinates without a magnetic field in the region from the Sun to the Earth's orbit. Protons are considered as particles while electrons are considered as a massless fluid, with a constant temperature, in order to study the relation between the hybrid and hydrodynamic solutions. It is shown that the strong electric field in the hybrid model accelerates the protons. Since the electric field in the model is related to electron pressure, each proton in the initial Maxwellian velocity distribution function moves under the same forces as in the classical Parker Solar wind model. The study shows that the hybrid model results in very similar velocity and number density distributions along the radial distance as in the Parker model. In the hybrid simulations, the proton temperature is decreased with distance in 1 order of magnitude. The effective polytropic index of the proton population slightly exceeds 1 at larger distances with the maximum value $\sim 1.15$ in the region near the Sun. A highly non-Maxwellian type of distribution function is initially formed. Further from the Sun, a narrow beam of the escaping protons is created which does not change much in later expansion. The results of our study indicates that already a nonmagnetized global hybrid model is capable of reproducing some fundamental features of the expanding solar wind shown in the Parker model and additional kinetic effects in the solar wind.

\section{Introduction}

The hydrodynamic description of the coronal plasma escape from the solar surface was first proposed by Parker [1958], which implied a polytropic relationship between the temperature and plasma density. This model has two important parameters: the polytropic index, $\gamma$, and the ratio of the gravitational and thermal energies $\lambda_{0}=G M m /\left(R_{0} k_{B} T_{0}\right)$, where $G$ is the gravity constant, $M$ is the mass of the Sun, $m$ is the mass of a particle, $k_{B}$ is the Boltzmann constant, and $R_{0}$ and $T_{0}$ are the radius and temperature of the lower reference boundary, respectively. Parker investigated solutions for different parameters $\left(\lambda_{0}, R_{0}\right)$ and his findings show that the physical solution, which starts at $R_{0}$ with a subsonic velocity is passing through the critical point and has to satisfy the following conditions:

$$
\gamma \leq 3 / 2, \quad 2 \gamma \leq \lambda_{0} \leq \gamma /(\gamma-1)
$$

In an isentropic flow case without heating, we have $\gamma=5 / 3$ for a monatomic gas. In such a case the conditions above are not satisfied and thus the subsonic flow cannot pass by a sonic point and cannot reach a supersonic velocity. Taking $\gamma$ to be less than $5 / 3$, we imply a distributed volume heating which is proportional to $(5 / 3-\gamma)$ [see Lamers and Cassinelli, 1999]. Condition (1) determines a lower limit for heating which can provide acceleration of the plasma flow from a subsonic velocity to a supersonic one. If condition (1) is not satisfied, then the flow should be supersonic even at the lower boundary. In a one-fluid hydrodynamic case, we do not have a force able to immediately accelerate the flow to the supersonic velocity just at the lower boundary. However, in a kinetic or hybrid model case the fast flow acceleration can be provided by a strong electric field in the vicinity of the lower boundary, as will be discussed later in the paper. After the Parker model, further developments in solar wind modeling were performed mainly in two directions: (1) two-fluid approach taking into account different temperatures of the protons and electrons [Sturrock and Hartle, 1966; Hartle and Sturrock, 1968] and also pressure anisotropy related to the magnetic field [Leer and Axford, 1972]; 
(2) kinetic description of the solar wind plasma in electric and gravitation fields. All fluid models assume some value of the effective polytropic index which has a crucial influence on the solution. In addition, the most advanced fluid models include energy and momentum sources due to absorbtion of the Alfven wave perturbations propagating from the Sun. Various modifications of the two-fluid models were considered by Cuperman and Harten [1970, 1971], Hartle and Barnes [1970], Habbal et al. [1995], Tu and Marsch [1997, 2001], Esser and Habbal [1995], and Kim et al. [2004]. Kinetic models pioneered by Chamberlain [1960] can provide more rich and detailed description of the solar wind flow compared to the fluid models. In particular, kinetic models may yield non-Maxwellian features of distribution functions, a radial electric field which is necessary to keep quasi-neutrality of the solar wind plasma in the gravitational field of the Sun [Lemaire and Scherer, 1973], and possibly can take into account effects of interaction between particles and plasma waves [Marsch, 2006]. Physical aspects and advances of the kinetic approach in application to the solar wind flow were discussed by Meyer-Vernet [2007] and Echim et al. [2011]. The advantage of kinetic models is that they allow one to obtain particle distribution functions which are rather different to derive from the Maxwellian ones. The main feature of the kinetic models is the appearance of a radial electric field, which is needed to provide a quasi-neutrality condition for plasma in the gravitational field. An additional condition to be fulfilled is that the radial electric current has to vanish. The existing kinetic models are based on steady state analytical solutions. Regarding this, a question arises about the stability of such solutions. A numerical hybrid approach has an intermediate status between the kinetic and fluid models, because it considers protons as particles and electrons as a fluid. This approach has been used earlier to study the expansion of the solar wind in a moving and expanding simulation box [see, e.g., Liewer et al., 1999; Hellinger et al., 2003; Tulasi et al., 2013; Parashar et al., 2013]. However, in this study we present a global Euler formalism hybrid numerical model for the simulation of the solar wind in spherical coordinates from the Sun to Earth's orbit.

\section{Model Description}

The adopted hybrid model is the part of the MULTI space plasma simulation platform which includes different hybrid models to study the interaction of various solar system bodies with the solar wind (Mars, Venus, the Moon, Saturnian satellite Titan, comets, asteroids, etc.). Recently, the original Cartesian mesh model was extended to the spherical mesh and inherits the main properties of the Cartesian platform. The model equations are described in detail in Kallio and Janhunen [2003] and its spherical mesh version in Dyadechkin et al. [2013].

If we suppose that there is no magnetic field, then the system of hybrid model equations can be reduced to the following set of equations:

$$
\begin{gathered}
n_{e}=n_{i} \\
\mathbf{E}=-\frac{\nabla p_{e}}{e n_{e}} \\
\frac{\mathrm{d} \mathbf{v}_{i}}{\mathrm{~d} t}=\frac{q_{i}}{m_{i}} \mathbf{E}+\frac{F_{\text {gravitation }}}{m_{i}} \\
\frac{\mathrm{d} \mathbf{r}_{i}}{\mathrm{~d} t}=\mathbf{v}_{i}
\end{gathered}
$$

Equation (2) denotes the quasi-neutrality condition, where $n_{e}$ and $n_{i}$ are the electron and ion number densities, respectively. The parameters $m_{i}, q_{i}, r_{i}$, and $v_{i}$ are the mass, electric charge, the position, and the velocity of ions, respectively. In the analyzed simulation the ions were assumed to be protons. Equation (3) describes the electric field, where $\nabla p_{e}$ is the gradient of the electron pressure and equation (4) Newton's second law including the electric force and the gravitational force $F_{\text {gravitation }}$.

The described system of equations is a closed system and describes the evolution of ion positions $\mathbf{x}_{i}$ and ion velocities $\mathbf{v}_{i}$ self-consistently from their initial state. In the model that is used, the particles are propagated with a leapfrog algorithm (see Kallio and Janhunen [2003] for the details of the algorithms). During a single time step, dt, the quantities are evaluated from $\left(\mathbf{x}_{i}^{t-1 / 2}, \mathbf{v}_{i}^{t}\right)$ to $\left(\mathbf{x}_{i}^{t+1 / 2}, \mathbf{v}_{i}^{t+1}\right)$. 
If we assume for simplicity that the electron temperature $T_{e}$ is a constant, we get from equation (3) the radial electric field, $E_{r}$ :

$$
E_{r}=-\frac{k_{B} T_{e}}{e n_{e}} \frac{\partial n_{e}}{\partial r}
$$

an equation which describes the ambipolar field in terms of the number density gradients.

If we consider only the radial motions of the particles, as is done in the developed hybrid model, we can therefore simplify the system of equations (2)-(5) rewriting them to a 1-D radial case:

$$
\begin{gathered}
n_{e}=n_{i}, \\
\frac{\mathrm{d} v_{r, i}}{\mathrm{~d} t}=\frac{1}{m_{i}}\left(-\frac{k_{B} T_{e}}{n_{e}} \frac{\partial n}{\partial r}\right)-\frac{G M}{r^{2}}, \\
\frac{\mathrm{d} r_{i}}{\mathrm{~d} t}=v_{r, i} .
\end{gathered}
$$

In our model we use the simulation domain which is based on the spherical coordinate grid [Dyadechkin et al., 2013]. The main difference between the full 3-D case and the analyzed case is that we consider a pseudo 1-D case and use only a single grid layer in $\theta$ and $\phi$ directions. In the simulation, the following spherical coordinate simulation box is used: $r=\left[r_{\text {min }}, r_{\max }\right], \theta=[\pi / 2-\pi / 24, \pi / 2+\pi / 24]$ and $\phi=[-\pi / 24, \pi / 24]$, where $r_{\text {min }}=10^{6} \mathrm{~km}$ and $r_{\max }=151 \times 10^{6} \mathrm{~km}$. The number of grid cells in three dimensions was $n_{r} \times n_{\theta} \times n_{\phi}=7500 \times 1 \times 1$. The radial cell size, $d r$, was $20,000 \mathrm{~km}$, and the number of macropatricles per cell was 300 . The simulation time step was $10 \mathrm{~s}$. The simulation time step, $\mathrm{dt}$, was chosen to be sufficiently small so that the majority of the protons do not jump over a cell during $\mathrm{d} t, \mathrm{~d} t / \mathrm{d} r=20,000 \mathrm{~km} / 10 \mathrm{~s}=2000 \mathrm{~km} / \mathrm{s}$, while the initial thermal velocity of the protons was $90 \mathrm{~km} / \mathrm{s}$ and the maximum bulk velocity of a proton in the simulation was about $600 \mathrm{~km} / \mathrm{s}$. To check how grid resolution affects the solution, were performed several runs with smaller and higher radial grid sizes. It turned out that the result does not depend on the spatial resolution within the computational noise limit.

Simulated particles, the so-called macroparticles, correspond to a certain number of real particles [see Dyadechkin et al., 2013] that only move along the radial line: $[\theta, \phi]=[\pi / 2,0]$. This means that the center of the macroparticle is always located on this line. We used an absorbing boundary condition for the particles, which is applied to the $R_{\min }$ and $R_{\max }$ surface. If the center of a macroparticle crossed the outer surface $\left(r_{\operatorname{mp}}^{\text {center }}>R_{\max }\right)$ or the inner surface $\left(r_{\mathrm{mp}}^{\text {center }}<R_{\mathrm{min}}\right.$ ), the macroparticle was removed from the simulation box. The radial electric field, $E_{r}$, is stored on the cell faces, and it is calculated at the particle position via linear interpolation.

\section{Results of the Numerical Simulations}

In this section we describe the results of the numerical simulations and compare the results with the Parker's solar wind model [Parker, 1958].

We used only one particle species, protons $\left(H^{+}\right)$, which were launched from the inner radius $r=R_{\min }$. These particles were generated within the first grid cell by using a Maxwellian velocity distribution function with proton temperatures of $T_{p}=10^{6} \mathrm{~K}$. The simulations were performed for three different electron temperatures: $T_{e, 1}=1.5 \times 10^{6} \mathrm{~K}, T_{e, 2}=2.0 \times 10^{6} \mathrm{~K}$, and $T_{e, 3}=3.0 \times 10^{6} \mathrm{~K}$. The number density, $n_{0}$, at the inner radius of the simulation box $r=R_{\min }$, was $10^{14} \mathrm{~m}^{-3}$, and the initial radial proton bulk velocity, $U_{r, 0}$, was zero. The simulation time is $3 \times 10^{6} \mathrm{~s}$.

There is a small relaxation time for the hybrid solution to reach steady state, the time scale of which is approximately the time it takes for the slowly moving protons to fill the simulation domain (see Figure 1). For example, the Earth's orbit relaxation time in the simulation is about 5 days. The data from the numerical simulations were taken after the solution reached the steady state regime.

The distribution of the bulk radial velocity and the number density along the radial distance from the Sun is presented in Figures 2 and 3, respectively. As can be observed the hybrid solution shows a noticeable agreement with Parker's isothermal model of the solar wind. 

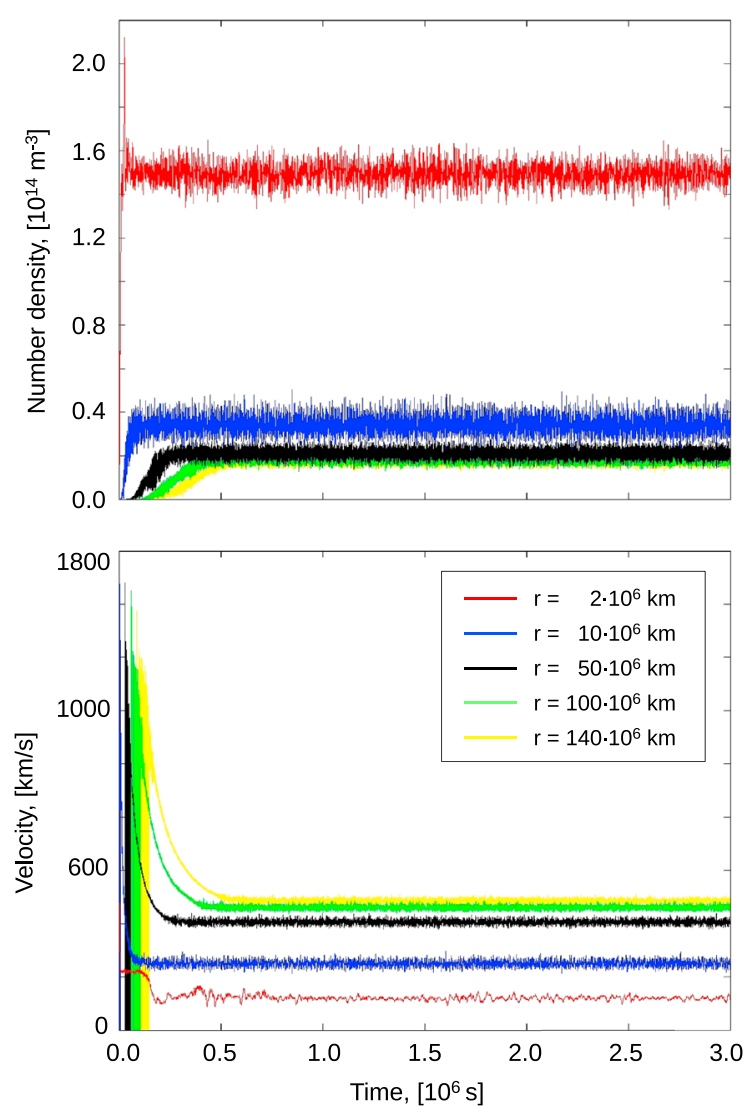

Figure 1. Time dependencies for (top) number density and (bottom) bulk velocity at three radial distances from the center of the Sun in the developed hybrid simulation. The figure demonstrates that the relaxation time within the simulation box is about $0.5 \times 10^{6} \mathrm{~s}$. The figure also shows the level of statistical fluctuation.

Since the radial profiles of the bulk velocity and the number density are very similar to the Parker's profiles, the total radially outward mass flux, $\rho_{m}\left(=m_{p} n U_{r} 4 \pi r^{2}\right)$, obtained from the hybrid simulations (see Figure 4) corresponds to the mass loss rate taken from the Parker model. Figure 4 shows that the mass flow is nearly constant along the radial distance with stronger noise for the higher electron temperature.

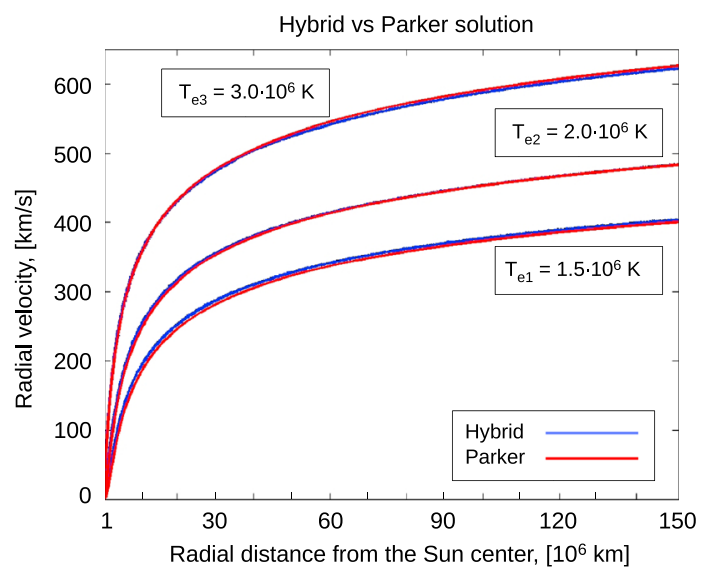

Figure 2. Bulk velocities, $U_{r}$, for three different electron temperatures with respect to the radial distance. The blue lines are the results of the hybrid simulation, and the red lines are the Parker solution for the isothermal solar wind for three different electron temperatures: $T_{e, 1}=1.5 \times 10^{6} \mathrm{~K}, T_{e, 2}=2.0 \times 10^{6} \mathrm{~K}$, and $T_{e, 3}=3.0 \times 10^{6} \mathrm{~K}$. The velocity profiles from the numerical simulations were taken when the solution reached the steady state regime. 


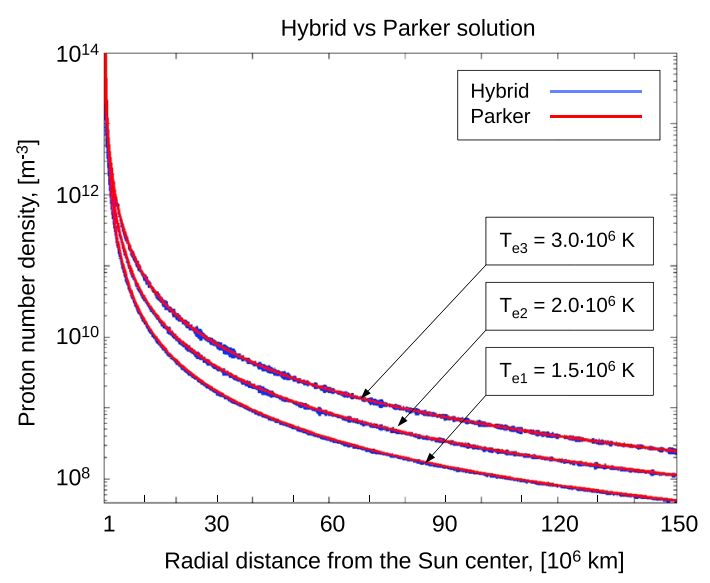

Figure 3. Number density distribution against the radial distance for three electron temperatures (blue lines) in the hybrid model. The red lines show the number densities for similar temperatures, which were calculated based on the Parker solution. The initial value of the number density for the Parker solution was the same as that used in the hybrid model simulation.

It should also be noted that although the bulk velocity, number density, and the mass loss rate in the hybrid model were similar to the isothermal Parker model, there is also an important difference between the models. Instead of a constant hydrodynamic temperature in the Parker's model, the electron and proton temperatures as in the hybrid simulations are different. The behavior of the proton temperature $T_{p}$ along the radial distance is shown in Figure 5. It can be seen that $T_{p}$ initially drops rapidly an order of magnitude and then decreases slowly with increasing distance from the Sun.

Such behavior can be explained in terms of the electric field acceleration. The potential energy associated with the electric potential, $\varphi_{E}$, is given in Figure 6. Furthermore, the change of the potential energy of the protons, which moves from the distance $r$ to the distance $r_{0}, \varphi_{g}$, is $\Delta \varphi_{g}(r)=\varphi_{g}(r)-\varphi_{g}\left(r_{0}\right)=G m_{p} M / r_{0}-G m_{p} M / r$ where $r_{0}=r_{\min }$. Since the electric field is associated in the hybrid model with the electron pressure (see equation (6)) change of the electric potential energy of a proton, $\varphi_{E}$, is

$$
\Delta \varphi_{E}(r)=\varphi_{E}(r)-\varphi_{E}\left(r_{0}\right)=k_{B} T_{e} \ln \frac{n_{e}}{n_{0}},
$$

where $n_{0}$ is the number density at the inner surface $r=r_{0}$. The electric potential energy decreases rapidly near the Sun and then continues to decrease slowly. As a consequence, the protons are initially accelerated by the strong electric field and then rapidly cooled when expanding into a vacuum. The protons are cooled at the same time when the initial Maxwellian velocity distribution function becomes non-Maxwellian when particles move through the total potential structure.

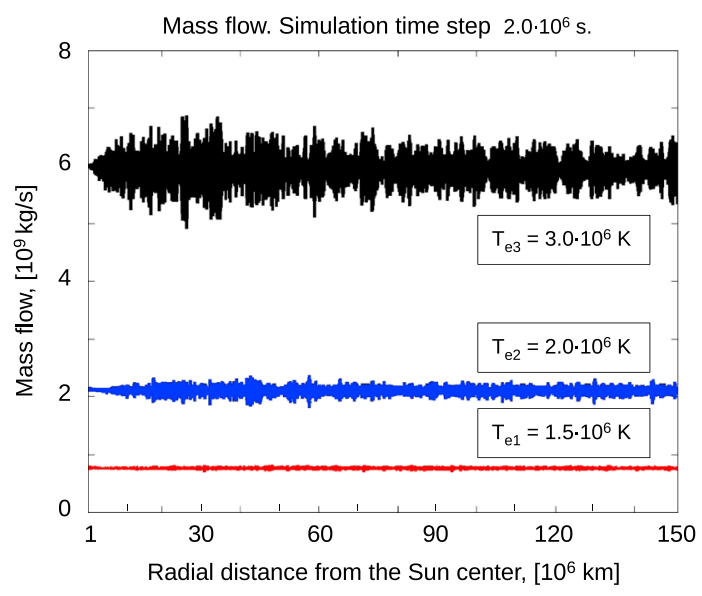

Figure 4. The mass flow flux, $m_{p} n U_{r} 4 \pi r^{2}(\mathrm{~kg} / \mathrm{s})$, for three different electron temperatures: red line for $T_{e, 1}=1.5 \times 10^{6} \mathrm{~K}$, blue line for $T_{e, 2}=2.0 \times 10^{6} \mathrm{~K}$, and black line for $T_{e, 3}=3.0 \times 10^{6} \mathrm{~K}$. The values are taken at $t=2 \times 10^{6} \mathrm{~s}$. 


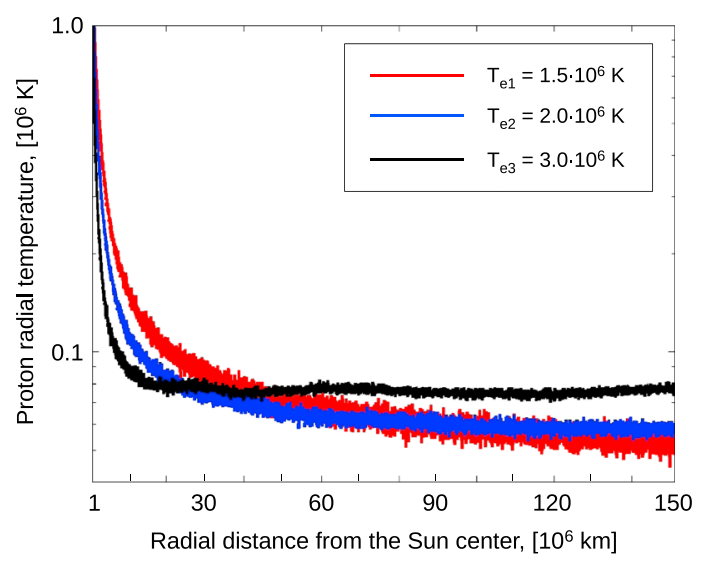

Figure 5. Profiles of the radial protons temperature $T_{p}$ for three different electron temperatures in the hybrid model: the red line: $T_{e, 1}=1.5 \times 10^{6} \mathrm{~K}$, the blue line: $T_{e, 2}=2.0 \times 10^{6} \mathrm{~K}$, and the black line: $T_{e, 3}=3.0 \times 10^{6} \mathrm{~K}$. The $T_{p}$ is derived from the protons pressure $T_{p}=P /\left(n k_{B}\right)$. The figure represents the time moment $t=2 \times 10^{6} \mathrm{~s}$ of the simulation.

As mentioned in section 1, the Parker model is very sensitive to the value of the polytropic index $\gamma$, which has to satisfy the condition $\gamma \leq 3 / 2$. It is interesting to find the effective polytropic index from our hybrid simulations. Using pressure variations $p$ and corresponding number density variations $n$, we can find the polytropic index as $\gamma=\ln \left(p / p_{0}\right) / \ln \left(n / n_{0}\right)$, where $n_{0}$ and $p_{0}$ are the number density and pressure values, respectively, at $r=R_{\max }$. The dependence of $\ln \left(p / p_{0}\right)$ on $\ln \left(n / n_{0}\right)$ is presented in Figure 7. As one can see in the figure, the higher the electron temperature, the closer the profile to the line $\gamma=1$. This can be seen more clearly in Figure 8 where the function $\gamma(r)$ is shown. The maximum value of $\gamma_{\max } \sim 1.15$ is found to be in the region near the Sun. The characteristic distances of $\gamma$ profiles look similar to the proton temperature profiles in Figure 5 . It seems that the scale of the polytropic index variation coincides approximately with the critical distance (or distance of the critical point) of the Parker model, $r_{c}=G M / c_{s}^{2}$, where $c_{s}=\sqrt{k T_{e} / m_{p}}$ is now the speed of the ion acoustic wave instead of the sound speed of the Parker model.

It should be noted that our estimation of the polytropic index $\gamma$ is slightly less than that obtained from the observation in the solar wind, which is not surprising, due to the simplicity of our model. For example, Sittler and Scudder [1980] estimated based on Voyager 2 and Mariner 10 data that $\gamma=1.17$, while Whang [1998] obtained $\gamma=1.28$ based on Voyager 2 data. In addition, Totten et al. [1995] derived the maximal value $\gamma=1.46$ based on Helios proton data.

Figure 9 presents the proton distribution function evolution in the course of the solar wind expansion obtained from the hybrid simulations. As can be seen in the figure, initially, the distribution function was

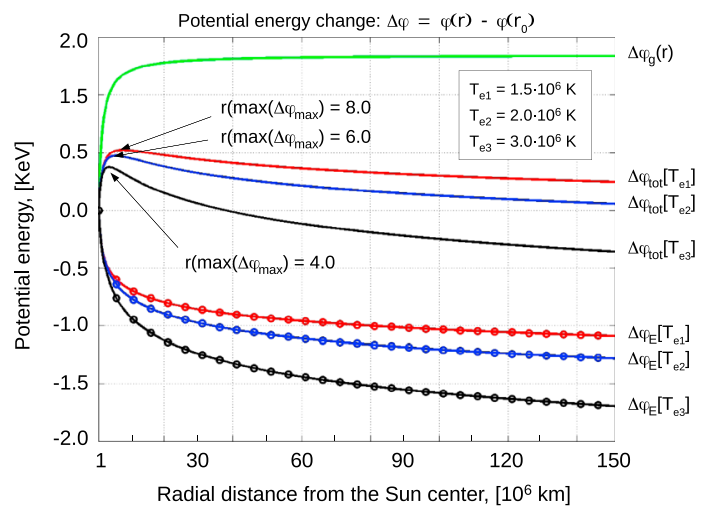

Figure 6. Profiles of the electrical (e $\varphi_{E}$, dotted lines), gravitational $\left(m_{p} \varphi_{g}\right.$, the solid green line), and the total (electrical + gravitational) potential energy change relative to the inner face $\left(r=R_{\min }\right)$ in the hybrid stimulation for three different electron temperatures. The solid red, blue, and black lines represent the electrical potential for $T_{e, 1}, T_{e, 2}$, and $T_{e, 3}$, respectively. The solid red, blue, and black lines which are marked by circles represent the total potentials for $T_{e, 1}, T_{e, 2}$, and $T_{e, 3}$, respectively. 


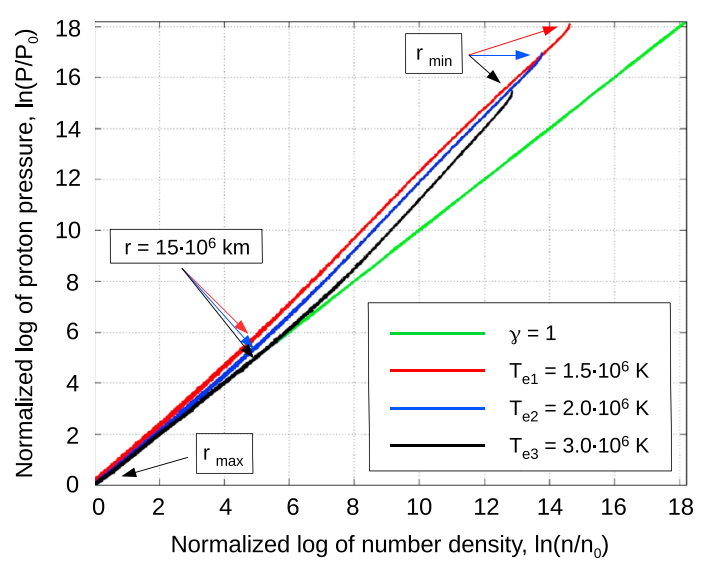

Figure 7. Profiles for adiabatic indexes for three different electron temperatures in the hybrid model: the red line for $T_{e, 1}=1.5 \times 10^{6} \mathrm{~K}$, the blue line for $T_{e, 2}=2.0 \times 10^{6} \mathrm{~K}$, and the black line for $T_{e, 3}=3.0 \times 10^{6} \mathrm{~K}$. The green line represents the case when the adiabatic index $\gamma=1$, and it is added to the figure for better comparison of the numerical results. The figure represents the time moment $t=2 \times 10^{6} \mathrm{~s}$ of the simulation. Pressure, $P$, and number density, $n$, normalized to $P_{0}$ and $n_{0}$ the values of pressure and number density, respectively, at $r=R_{\max }$.

chosen to be Maxwellian at the Sun's boundary of the simulation domain. Then the protons start to be accelerated outward by the electric field, which leads to the formation of the proton beam. The first departure stage of the beam formation is clearly seen for the range $\mathrm{d} r=\left[1 \times 10^{6}, 21 \times 10^{6}\right] \mathrm{km}$ in Figure 9a. The leading part of the distribution function consists of the escaping protons, the trailing plateau-like part being populated with the ballistic particles which do not have enough energy to overtake the potential barrier (see Figure 6) and which are reflected back. The next stage of the velocity distribution function evolution is shown for the range $\mathrm{d} r=\left[21 \times 10^{6}, 41 \times 10^{6}\right] \mathrm{km}$. Here one can see only the beam of the escaping protons. The distribution function becomes more and more narrow with the increasing radial distance. Note that the distribution function continues to be non-Maxwellian in the simulation, as can be seen by noting that the velocity distribution function remains asymmetric with respect to the mean speed $v_{\mathrm{SW}}$.

It is worth noting that due to the conservation of angular momentum, $v_{\phi r}=$ const, the distribution function becomes narrow very soon with respect to transverse velocity components. The relation $v_{\phi r}=$ const leads to the fact that the width of distribution function decreases inversely proportional to the distance from the Sun. Therefore, we consider only radial particle motion.

Although we concentrated in this paper on steady state solution, it is still worth to show the formation of the supersonic proton flow. The solar wind is formed by emitting protons (better to say macroparticles) from

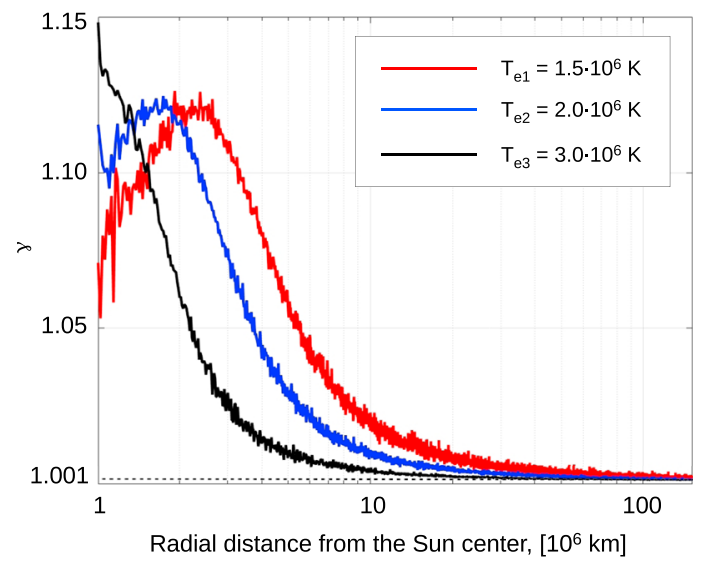

Figure 8. The radial dependence of the polytropic index, $\gamma(r)$, for three different electron temperatures in the hybrid model at different distances from the center of the Sun: the red line for $T_{e, 1}=1.5 \times 10^{6} \mathrm{~K}$, the blue line for $T_{e, 2}=2.0 \times 10^{6} \mathrm{~K}$, and the black line for $T_{e, 3}=3.0 \times 10^{6} \mathrm{~K}$. The figure represents the time moment $t=2 \times 10^{6} \mathrm{~s}$ of the simulation. 


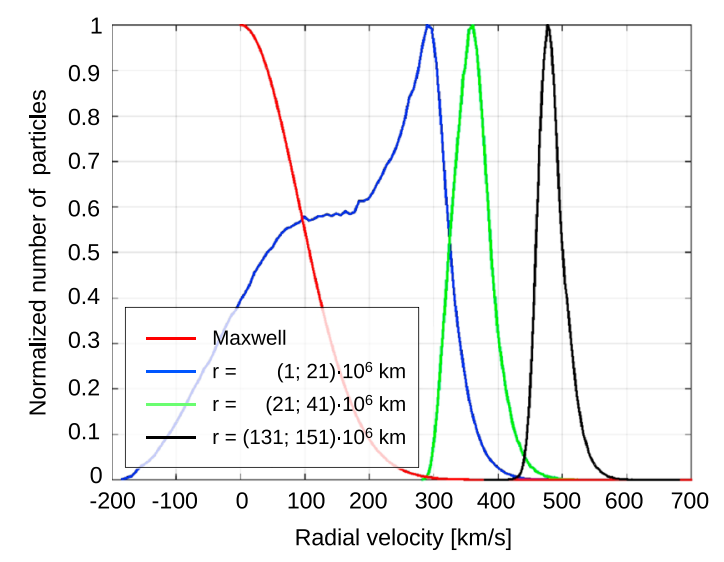

Figure 9. (top) Demonstration of the velocity distribution function for three different spatial ranges $d r$ in the hybrid model: the blue line shows the velocity distribution for $d r_{1}=\left(1 \times 10^{6}, 21 \times 10^{6}\right) \mathrm{km}$, the green line shows the velocity distribution for $d r_{1}=\left(21 \times 10^{6}, 41 \times 10^{6}\right) \mathrm{km}$, and the black like shows the velocity distribution for $d r_{1}=\left(131 \times 10^{6}\right.$, $\left.151 \times 10^{6}\right) \mathrm{km}$. All profiles are normalized to the maximum number of particles for each profile. The red line represents the positive part, i.e., outward moving particles of the Maxwellian velocity distribution function for which the initial bulk velocity was zero and the initial temperature of the protons was $10^{6} \mathrm{~K}$. The figure represents the time moment $t=2 \times 10^{6} \mathrm{~s}$ of simulation.

the Sun-faced side wall of the simulation box. Macroparticles are emitted with the Maxwellian distribution function, so there is a number of particles with high thermal velocities from the tail of the distribution, which propagate far away from the wall even for one simulation time step. These particles form the leading front of the wind and create the initial gradient of number density, which in its turn leads to generation of the electric field (see equation (6)). Electric field is derived as a cell center value according to number density distribution. At the initial stage when the macroparticle has not yet filled up the whole simulation box, it is possible that inside a grid cell there are not even a single macroparticle. Therefore, electric field can be infinitely large because the number density of the denominator in equation (6) is zero. To prevent this situation, we use background electron number density and insert it into a cell in which the electron number density is lower than some critical value. This background critical electron number density in our simulation was set to be $10^{5} \mathrm{~m}^{-3}$, while the macroparticle weight (number of protons in single macroparticle) is $8 \cdot 10^{33}$, which gives number density of the order of $10^{9} \mathrm{~m}^{-3}$. The electric field produced by the gradient of number density accelerates particles. As a result of this additional acceleration and initial thermal particles, the leading extending region is formed with supersonic flow behind. The maximum value of the flow is mainly defined by the initial thermal velocity which is larger than both the speed of the ion acoustic wave and steady state speed of the wind (Figure 10). In the course of time the leading region is propagating outward the Sun, and slowly, the flow velocity tends to its steady state value.

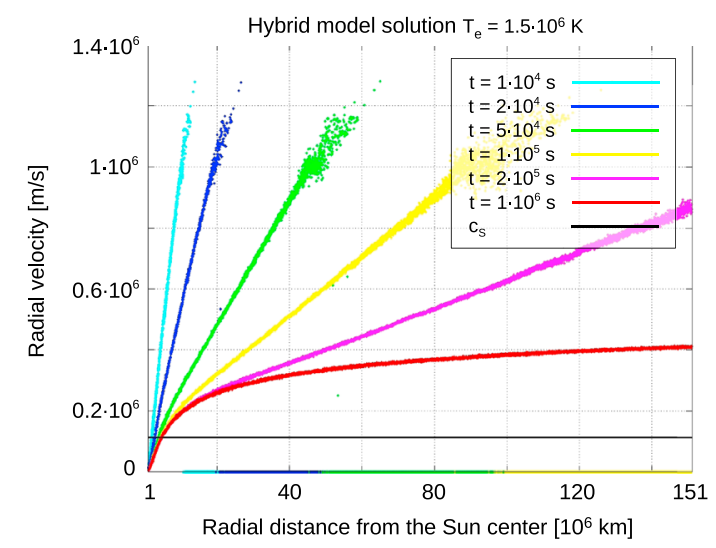

Figure 10. Illustration of time dependency of radial bulk velocity $V_{r}$ during different stage of simulation. Different colors represent different time moments as mentioned on the figure expositor. The red line corresponds to the beginning of the steady state regime $\left(t=10^{6} \mathrm{~s}\right)$. Electron temperature in the presented case is $T_{e, 1}=1.5 \times 10^{6} \mathrm{~K}$. 


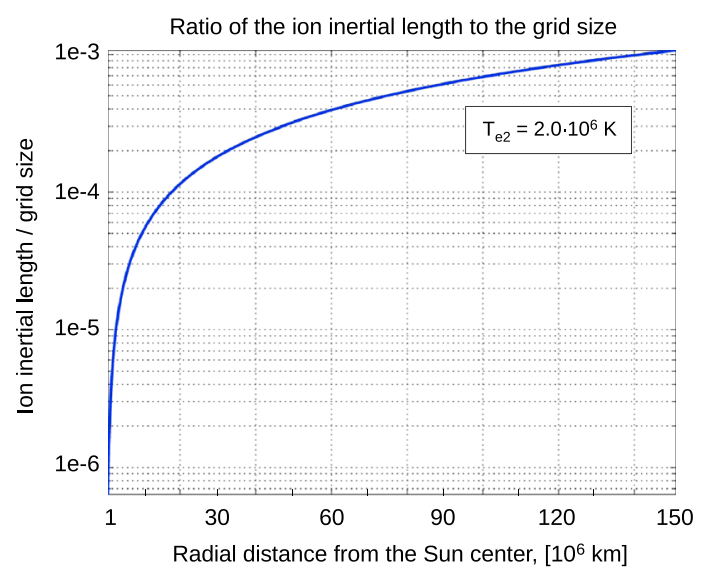

Figure 11. The ratio of the ion inertial length to the grid size as function of the radial distance. Electron temperature in the presented case is $T_{e, 2}=2.0 \times 10^{6} \mathrm{~K}$.

At the end of this section it is worth to mention the limitation of the used simulation grid. Figure 11 shows ratio of the inertial length to the grid size as a function of the radial distance. This ratio is rather small and thus the grid size much exceeds the inertial length scale. Comparison of the numerical solutions obtained for different grid sizes indicates that the resolution is sufficient for the gradient scales. But one has to keep in mind that our grid size is not sufficient to resolve possible plasma instabilities, which can appear due to a particular shape of the ion distribution function. However, usage of very fine grid size in the whole large-scale calculation domain would require too large computational capacities. An alternative way is to identify possible unstable regions using the previously obtained large-scale solution. Therefore, we would consider the aspects of possible instabilities as a subject for future study.

\section{Discussion}

This study describes, according to the best of the authors' knowledge, the first detailed analysis of a global spherical symmetric kinetic hybrid model and its relation to the classical Parker model. In the kinetic model the radially expanding solar wind was assumed to be nonmagnetized, as was assumed in the Parker model.

The hybrid approach in our case can be considered as a two-fluid model with the pressure established by the electrons through the electric field and also by the protons through the second moment of the distribution function. As it was pointed out by Sturrock and Hartle [1966] who made two-fluid modeling of the solar wind, the important feature of the two-fluid models is that the electron temperature is usually higher than the proton temperature; hence, the electron pressure dominates. In our extreme hybrid model case, where electrons are massless particles and their temperature was kept constant, the proton temperature is reduced drastically more than 1 order of magnitude (see Figure 5). This means that proton pressure is not significant any more in the equation of motion. Hence, in the first approximation after neglecting the proton thermal pressure, the hybrid approach under consideration can be interpreted as a variant of the proton one-fluid hydrodynamics but with the pressure established by the electrons through the electric field. Moreover, the resulting system of equations is formally identical to the system which Parker used for his solution. That is why our results for the proton velocity, number density, and mass loss rate (but not for the proton temperature) are very close to the Parkers solution. Nevertheless, the physics of both solutions is rather different: the Parker solar wind is accelerated by the pressure gradient of the initially heated gas, while in the hybrid approach the solar wind is accelerated by electric field produced by the electrons.

The hybrid approach also reveals important differences from the Parker model. First of all, the proton temperature decreases by more than 1 order of magnitude due to electric field acceleration. Second, we were able to find the effective polytropic index for the proton gas that turns out to be a function of radial distance with the maximum value $\gamma_{\max } \sim 1.15$. Variations in both the proton temperature and the polytropic index had the length scales of several $\left(2 r_{c}-3 r_{c}\right)$ critical distances of the Parker model.

In the simulation, electrons are kept at a constant high temperature without heat flux and heating. This assumption is based on the very high ratio of the electron and ion thermal conductivities. As was shown by 
Sturrock and Hartle [1966] for the two-fluid solar wind model, the variation of electron temperature is much smaller than that of ions. Therefore, a simplified assumption of a constant electron temperature as a first step for hybrid simulation was adopted. This assumption was also appropriate for comparison with the Parker solution. The next step in the development of the simulation would require energy equation and temperature anisotropy of electrons depending on the magnetic field.

In the region near the Sun, we observed particles which have different orbits: escape, ballistic with a plateaulike distribution function. Further from the Sun, these ballistic particles disappear and eventually a beam of protons is created, with the distribution function remaining non-Maxwellian in the hybrid model. Further investigation to the asymmetry with respect to Kappa distributions observed in the solar wind (as reviewed by Pierrard and Lazar [2010]) can be foreseen. The electric field at its strongest near the Sun and the total potential energy difference is about equal to the gravitational potential energy. This left the escaping particles sufficient energy to overcome the gravitational barrier and escape from the Sun.

It is interesting to note that the manner in which the solar wind protons escape from the Sun's gravitational field has certain phenomenological similarities with how the photoelectrons can escape from the surface of an airless object: in both cases outflowing particles have to overcome a local potential barrier after which they can escape from the object [see Dyadechkin et al., 2015].

Coulomb collisions are neglected in the developed simulation. As pointed out by Marsch and Goldstein [1983], the high-speed solar wind ion distributions look like collisionless plasma. However, for low-speed solar wind one often finds nearly isotropic ion distributions, which can be related to the Coulomb collisions. Therefore, for further applications of the hybrid model to the slow wind, it would be important to take into account also Coulomb scattering of ions.

Here we demonstrate only steady state results of solar wind propagation and we use only an initial Maxwellian distribution function for velocities. However, the developed kinetic model allows us to start our simulations initially with an arbitrary velocity distribution function, several ion populations, (e.g., fast and slow winds), multiple ion species, (e.g., $\mathrm{He}^{++}$) and multiple-charged heavy ions. The time-dependent model also gives us a possibility to simulate dynamical processes in the solar wind such us the number density or velocity jumps (then the values of velocity or number density are increased on the inner boundary) and study their evolution, that can mimic interplanetary coronal mass ejections.

The kinetic model can also simulate 2-D and 3-D problems which are, however, computationally highly expensive and beyond the scope of the present study. Overall, the study suggests that already a nonmagnetized global hybrid model is capable of reproducing some fundamental features of the expanding solar wind, or stellar wind, shown in the Parker model. In addition, the new simulations require kinetic effects when the initial Maxwellian velocity distribution plasma becomes non-Maxwellian, the electrons to be considered nonisothermal, and simulation grid structure is nonuniform with implementation inside boundary layers.

Acknowledgments

N.V.E. acknowledges RFBR grants 15-05-00879-a and 16-52-14006 ANF_a, V.S.S. was supported by RFBR grant 16-05-00470. H.L. and N.V.E. acknowledge the support by the Austrian Research Foundation FWF NFN projectS11601-N16 "Pathways to Habitability: From Disk to Active Stars, Planets and Life" and the related subproject S11607-N16 "Particle/ Radiative Interactions with Upper Atmospheres of Planetary Bodies Under Extreme Stellar Conditions". The data presented in this paper are available upon request from the authors (S.D.).

\section{References}

Chamberlain, J. W. (1960), Interplanetary gas. II. Expansion of a model solar corona, Astrophys. J., 131, 47, doi:10.1086/146805. Cuperman, S., and A. Harten (1970), Noncollisional coupling between the electron and the proton components in the two-fluid model of the solar wind, Astrophys. J., 162, 315, doi:10.1086/150656.

Cuperman, S., and A. Harten (1971), The electron temperature in the two-component solar wind, Astrophys. J., 163(1), 383, doi:10.1086/150778.

Dyadechkin, S. A., E. Kallio, and R. Jarvinen (2013), A new 3-D spherical hybrid model for solar wind interaction studies, J. Geophys. Res. Space Physics, 118, 5157-5168, doi:10.1002/jgra.50497.

Dyadechkin, S., E. Kallio, and P. Wurz (2015), New fully kinetic model for the study of electric potential, plasma and dust above lunar landscapes, J. Geophys. Res. Space Physics, 120, 1589-1606, doi:10.1002/2014JA020511.

Echim, M., J. Lemaire, and O. Lie-Svendsen (2011), A review on solar wind modeling: Kinetic and fluid aspects, Surv. Geophys., 32(1), 1-70, doi:10.1007/s10712-010-9106-y.

Esser, R., and S. R. Habbal (1995), Coronal heating and plasma parameters at 1 AU, Geophys. Res. Lett., 22(19), 2661-2664, doi:10.1029/95GL02339.

Habbal, S. R., R. Esser, M. Guhathakurta, and R. R. Fisher (1995), Flow properties of the solar wind derived from a two-fluid model with constraints from white light and in situ interplanetary observations, Geophys. Res. Lett., 22(12), 1465-1468, doi:10.1029/95GL01064.

Hellinger, P., P. Trávniček, A. Mangeney, and R. Grappin (2003), Hybrid simulations of the expanding solar wind: Temperatures and drift velocities, Geophys. Res. Lett., 30(5), 1211, doi:10.1029/2002GL016409.

Hartle, R. E., and A. Barnes (1970), Nonthermal heating in the two-fluid solar wind model, J. Geophys. Res., 75(34), 6915-6931, doi:10.1029/JA075i034p06915.

Hartle, R. E., and P. A. Sturrock (1968), Two-fluid model of the solar wind, Astrophys. J., 151, 1155, doi:10.1086/149513.

Kallio, E., and P. Janhunen (2003), Modelling the solar wind interaction with Mercury by a quasineutral hybrid model, Ann. Geophys., 21(11), 2133-2145, doi:10.5194/angeo-21-2133-2003. 
Kim, S. J., K. S. Kim, Y. J. Moon, K. S. Cho, and Y. D. Park (2004), Numerical calculation of two fluid solar wind model, J. Korean Astron. Soc., 37(12), 55-59, doi:10.5303/JKAS.2004.37.1.55.

Leer, E., and W. I. Axford (1972), A two-fluid solar wind model with anisotropic proton temperature, Sol. Phys., 23(1), 238-250, doi:10.1007/BF00153907.

Lamers, H. J. G. L. M., and J. P. Cassinelli (1999), Introduction to Stellar Winds, Cambridge Univ. Press, Cambridge, U. K.

Lemaire, J., and M. Scherer (1973), Kinetic models of the solar and polar winds, Rev. Geophys., 11(2), 427-468, doi:10.1029/RG011i002p00427.

Liewer, P., M. Velli, and B. E. Goldstein (1999), Hybrid simulations of wave propagation and ion cyclotron heating in the expanding solar wind, Space Sci. Rev., 87, 257-260, doi:10.1063/1.58671.

Marsch, E. (2006), Kinetic physics of the solar corona and solar wind, Living Rev. Sol. Phys., 3(1), 1-100, doi:10.12942/Irsp-2006-1.

Marsch, E., and H. Goldstein (1983), The effects of Coulomb collisions on solar wind ion velocity distributions, J. Geophys. Res., 88(2), 9933-9940, doi:10.1029/JA088iA12p09933.

Meyer-Vernet, N. (2007), Basics of the Solar Wind, Cambridge Univ. Press, Cambridge, U. K.

Parashar, T. N., V. Marco, and B. E. Goldstein (2013), Expansion effects on solar wind hybrid simulations, AIP Conf. Proc., 1539, 54-57, doi:10.1063/1.4810988.

Parker, E. N. (1958), Dynamics of the interplanetary gas and magnetic fields, Astrophys. J., 128, 664-676.

Pierrard, V., and M. Lazar (2010), Kappa distributions: Theory and applications in space plasmas, Sol. Phys., 267(1), 153-174, doi:10.1007/s11207-010-9640-2.

Sittler, E. C., Jr., and J. D. Scudder (1980), An empirical polytrope law for solar wind thermal electrons between 0.45 and 4.76 AU-Voyager 2 and Mariner 10, J. Geophys. Res., 85, 5131-5137, doi:10.1029/JA085iA10p05131.

Sturrock, P. A., and R. E. Hartle (1966), Two-fluid model of the solar wind, Phys. Rev. Lett., 16(14), 628-631, doi:10.1103/PhysRevLett.16.628.

Totten, T. L., J. W. Freeman, and S. Arya (1995), An empirical determination of the polytropic index for the free-streaming solar wind using HELIOS 1 data, J. Geophys. Res., 100(A1), 13-17, doi:10.1029/94JA02420.

Tu, C. Y., and E. Marsch (1997), Two-fluid model for heating of the solar corona and acceleration of the solar wind by high-frequency Alfven waves, Sol. Phys., 171(2), 363-391, doi:10.1023/A:1004968327196.

Tu, C. Y., and E. Marsch (2001), Expansion effects on solar wind hybrid simulations, J. Geophys. Res., 106(A5), 8233-8252, doi:10.1029/2000JA000024.

Tulasi, P. N., M. Velli, and B. E. Goldstein (2013), Expansion effects on solar wind hybrid simulations, AIP Conf. Proc., 1539, 54-57, doi:10.1063/1.4810988.

Whang, Y. C. (1998), Solar wind in the distant heliosphere, J. Geophys. Res., 103(A8), 17,419-17,428, doi:10.1029/98JA01524. 